\title{
Markov-Based Lane Positioning Using Intervehicle Communication
}

\author{
Thanh-Son Dao, Keith Yu Kit Leung, Christopher Michael Clark, Member, IEEE, and Jan Paul Huissoon
}

\begin{abstract}
The majority of today's navigation techniques for intelligent transportation systems use Global Positioning Systems (GPS) that can provide position information with bounded errors. However, due to the low accuracy that is experienced with standard GPS, it is difficult to determine a vehicle's position at lane level. Using a Markov-based approach based on sharing information among a group of vehicles that are traveling within communication range, the lane positions of vehicles can be found. The algorithm's effectiveness is shown in both simulations and experiments with real data.
\end{abstract}

Index Terms-Global Positioning System (GPS), lane determination, lane-level positioning, Markov localization, vehicle navigation.

\section{INTRODUCTION}

I NTELLIGENT transportation systems are being developed in many different countries with the objective of improving road traffic efficiency and safety. Many systems have been proposed, and a large number of transportation research applications would benefit from a lane-level positioning capability. For example, such a capability could be used for a lane departure warning/lane keeping safety system in which an alarm will activate or autonomous driving will take over if the vehicle deviates from the center of the lane. Another application for lane-level positioning is a lane-level navigation system, which advises the driver as to which lane should be chosen to reach the specified destination without requiring excessive last-minute lane changing. Another approach is to use probe vehicles that measure lane-specific traffic conditions on a freeway. This is particularly important when understanding the efficiency of weaving road sections that may unnecessarily cause recurrent congestion [1].

Due to the importance of such applications, much effort has been put in lane finding/positioning, and commercially available lane finding/detection systems are already appearing. Ieng et al. [2] dealt with the multilane detection using multiple cameras. McCall and Trivedi [3] proposed a "video-based lane estimation and tracking" system, which was designed using

Manuscript received October 22, 2006; revised May 11, 2007, August 18, 2007, and August 22, 2007. This work was supported by the Automobile of the 21st Century (AUTO21). The Associate Editor for this paper was A. Eskandarian.

T.-S. Dao, K. Y. K. Leung, and J. P. Huissoon are with the Department of Mechanical and Mechatronics Engineering, University of Waterloo, Waterloo, ON N2L 3G1, Canada (e-mail: tsdao@engmail.uwaterloo.ca; kykleung@ lair.uwaterloo.ca: jph@mecheng1.uwaterloo.ca).

C. M. Clark is with the Department of Computer Science, California Polytechnic State University, San Luis Obispo, CA 93407 USA (e-mail: cmclark@ csc.calpoly.edu).

Digital Object Identifier 10.1109/TITS.2007.908574 steerable filters for robust and accurate lane-marking detection. Pierre-Yves and Jeff [4] used lane-level navigation systems with a high-level differential Global Positioning System (DGPS)/dead reckoning sensor integration system and a map database. Their system was able to detect which lane a car was driving in and when a car was changing lanes. Another approach [5] integrated an Inertial Measurement Unit (IMU) with a Global Positioning System (GPS) receiver to allow for accurate vehicle positioning. This approach also used a real-time kinematic DGPS receiver supported by DGPS base stations at the test site and a lane-level-detailed digital map. Inspired by the aforementioned approach, Wang et al. [6] developed a robust lane-departure warning system by equipping their Mercedes S-class with both a vision system and a high-precision GPS/Inertial Navigation System. Other research directions can be found in [7]-[11].

Most of these systems required image processing algorithms and/or costly equipment such as highly accurate sensors, highperformance computers, etc. With that in mind, we approach the problem by using intervehicle communication (IVC) to determine lane positions. IVC and cooperative driving systems have been under development for some time [12]-[15]. With the availability of GPS systems, it is practical to locate a vehicle with certain accuracy. However, GPS data do not provide the exact positions of vehicles (sometimes even placing the vehicle beside the road) due to degradation or multipath problems. Therefore, it is challenging to determine the exact lane that a vehicle is traveling in, even when a digital map of the road network is available.

In this paper, we describe our recent research in the area of low-cost lane-level position determination that can support a large number of transportation applications. A Markov-based approach, which computes the lane position of transport vehicles such as cars, buses, etc., is proposed. Our fundamental assumption is the existence of a number of vehicles equipped with GPS receivers. Each vehicle has the ability to communicate with other vehicles within a certain radius and is able to send its information regarding position to other vehicles via an ad hoc network. To support IVC, an IEEE 802.11p standard [16], [17] [also referred to as Wireless Access for the Vehicular Environment (WAVE)] was developed and could guarantee a maximum communication range of up to $1000 \mathrm{~m}$ under optimal conditions, or around $300 \mathrm{~m}$ for cars traveling at $200 \mathrm{~km} / \mathrm{h}$. Moreover, a new family of standards, referred to as IEEE 1609 suite [18], specifically for IVC built on IEEE 802.11 chipset is now under development. Three of the standards (i.e., IEEE Std. 1609.1 for Resource Management, IEEE Std. 1609.2 for Security Services for Applications and Management Messages, 
and IEEE Std. 1609.4 for Multichannel Operation) in the suite have been approved for trial use, and one (IEEE Std. 1609.3 for Networking Services) is pending. Once widely adopted, these standards will ensure that cars will have a communication range of 300-500 $\mathrm{m}$ on highways. Emergency vehicles will be equipped with longer ranged (1-km) WAVE systems. For our lane-positioning system, a conservative communication range estimate of $200 \mathrm{~m}$ is used for simulations and experiments, demonstrating that our system is realizable in future highway systems.

This paper is organized as follows. Section II gives a short introduction to GPS and the architecture of the lane-positioning system. Section III deals with the Markov localization algorithm in detail and its specific application to the lanepositioning problem. The simulation and experimental results are discussed in Sections IV and V, and some concluding remarks are provided in Section VI.

\section{GPS}

The GPS is a convenient method for determining vehicle position in a global coordinate system. It does this by using satellites and receivers. There are currently 24 satellites in orbit, which are operated by the U.S. Department of Defense, that provide worldwide coverage 24 hours a day, 7 days a week, in all weather. A detailed description of GPS is provided in [19]. In this system, the satellites send information to receivers, including time, $(x, y, z)$ position, and satellite signal strength among other things. The receivers obtain information from at least four satellites and use this to determine the receivers' $(x, y, z)$ location according to various measurements of the pseudorange between the satellite and the receiver antenna. The 2-D positioning accuracy offered by stand-alone GPS is $13 \mathrm{~m}$ (global average, 95\%) and $36 \mathrm{~m}$ (worst site, 95\%). Therefore, determining which lane a vehicle is traveling in is difficult using only GPS data from one vehicle.

Also, according to Grewal et al. [19], an error from GPS generally comes from several sources, including satellite clock, ephemeris error, ionospheric effects, tropospheric effects, and the geometry of visible satellites. This set of errors from GPS will be common for vehicles. Other errors are local to the different receivers and include radio frequency noise from the environment, receiver noise and resolution, multipath, and receiver clock error. Aside from multipath, errors from this second set are generally smaller than those common to the receivers. Hence, if multipath is not a problem (as is the case for many open highways), then receivers that are relatively close to one another will experience a similar GPS error. Although the absolute vehicle positions might have considerable errors, the relative position between vehicles should have a much lower error since many of the errors are common to the receivers. This is similar to what happens with DGPS, where the GPS solution is improved by removing the common errors. With this in mind, the relative distance between GPS measurements of vehicles can be utilized to estimate their lane positions.

While GPS measurements can be very precise in some circumstances, they generally are not accurate enough in rural areas where infrastructure for DGPS is not available, making lane position determination difficult if only GPS data from one vehicle are used. Even for future Geographic Information Systems (GIS) when information might be much improved to the point of determining where lanes are, it is still not clear to what availability this GIS information will be available. With an improved accuracy in mapping, there could be increased costs for access. Moreover, it is unclear how often this information will be updated to include changes in the roadway, including construction and traffic accidents, which divert traffic along different paths than recorded in the GIS database.

In this paper, it will be shown that by sharing state estimates between vehicles that are traveling close to each other, the lanelevel position for each vehicle can be determined. Fig. 1(a) illustrates the communication of vehicles on highway. Vehicles 1 and 2 are within communication range, and they can interchange information with each other, whereas vehicle 3 cannot communicate with vehicle 1 . The architecture for the system is shown in Fig. 1(b). Each vehicle is equipped with a GPS receiver and a processor to implement the lane-positioning algorithm and to communicate with other vehicles. The GPS data for the vehicles can also be fed into a position filter to reject the measurement noise from receivers. In our simulations and experiments, a combination of a particle filter [20]-[22] fused with a Butterworth filter [23] (other types of filters could also be used) can satisfy this task. With the number of particles of 500 being used, the processing time of the filtering algorithm only takes on the order of around 60-80 ms with an Intel Pentium-M $1.60-\mathrm{GHz}$ processor. Considering that the sampling rates of the GPS receivers are normally between 1 and $5 \mathrm{~Hz}$, the filtering algorithm can work well in real time.

\section{MARKOV LOCALIZATION}

Markov localization addresses the problem of state estimation from sensor data. Markov localization is a probabilistic algorithm: instead of maintaining a single hypothesis as to which lane the vehicles might be in, Markov localization maintains a probability distribution over the space of all such hypotheses. The probabilistic representation allows it to weight these different hypotheses in a mathematically sound way.

The target of Markov localization within this work is to determine which vehicle is traveling in which lane from GPS data using IVC. To introduce the major concepts of Markov localization, let us begin with a simple case, followed by a mathematical derivation of the algorithm. The reader may notice that Markov localization is a special case of probabilistic state estimation [24]-[26].

Let us start with the simplest case: two vehicles traveling on a two-lane road. The two vehicles are assumed close enough to be able to communicate with each other. Within this Markov localization approach, call $P\left(v_{1, t}=l_{a}, v_{2, t}=l_{b}\right)$ the probability that vehicle 1 is traveling in lane $a$ and vehicle 2 is in lane $b$ at time $t$, where $a$ and $b$ are either 1 or 2 . There are four possibilities in total, which are $P\left(v_{1}=l_{1}, v_{2}=l_{1}\right), P\left(v_{1}=\right.$ $\left.l_{1}, v_{2}=l_{2}\right), \quad P\left(v_{1}=l_{2}, v_{2}=l_{1}\right)$, and $P\left(v_{1}=l_{2}, v_{2}=l_{2}\right)$. These probabilities are equally initialized with 0.25 since no 


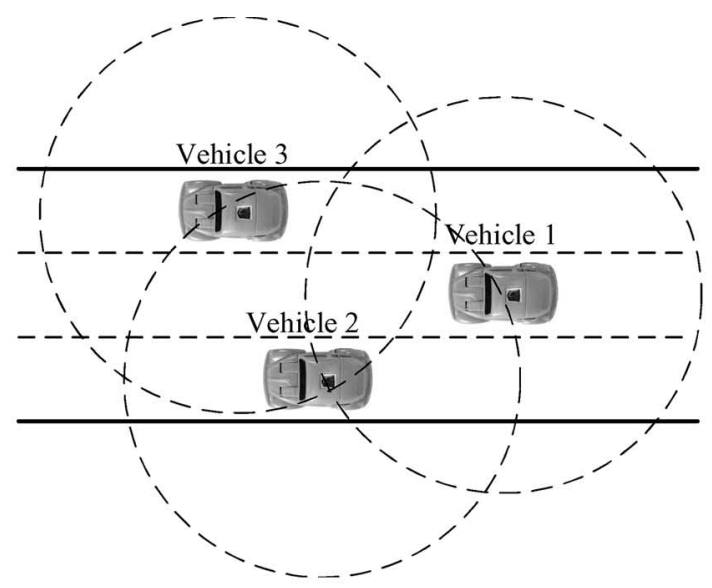

(a)
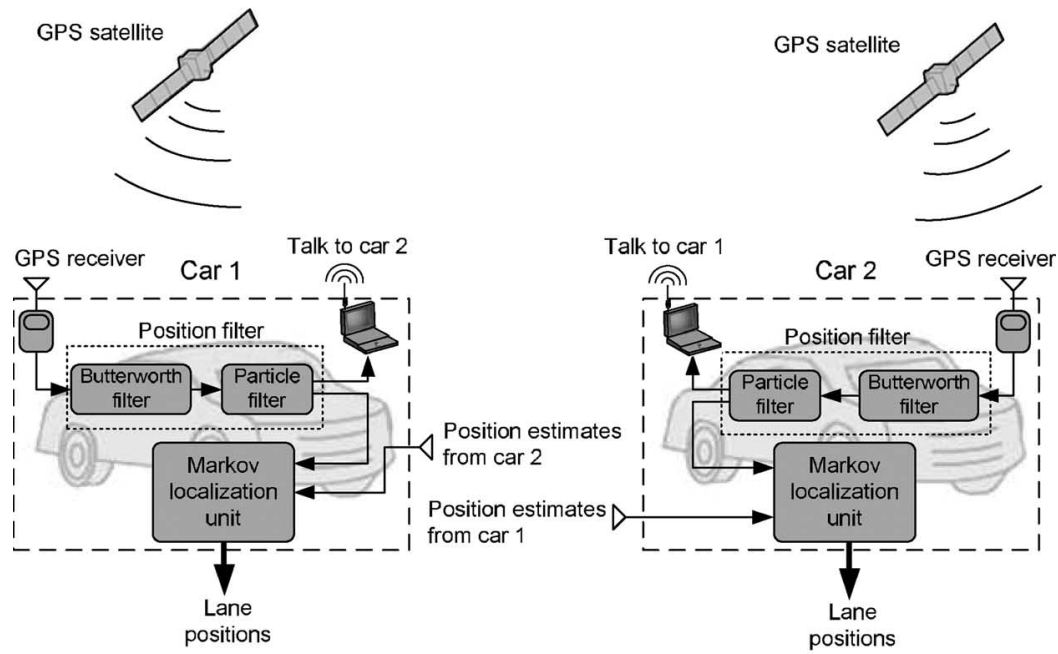

(b)

Fig. 1. (a) IVC (circles indicate the vehicles' effective radius of communication). (b) Architecture for the lane-positioning system.

initial estimates are required. In the course of the vehicles' mission (i.e., for $t>0$ ), $P$ is updated through two basic steps: 1) prediction and 2) correction.

\section{A. Prediction}

In the prediction step, the state of a vehicle is modeled through the conditional probability $P\left(v_{i, t}=l_{a} \mid v_{i, t-1}=l_{j}\right)$, which denotes the probability for a motion action that carries vehicle $i$ from lane $j$ to lane $a$ ( $j$ and $a$ can be equal). When the vehicle moves, $P\left(v_{i, t}=l_{a} \mid v_{i, t-1}=l_{j}\right)$, which models the uncertainty in the vehicle's dynamics, is used to compute the probability distribution at time $t$ as

$P\left(v_{i, t}=l_{a}\right) \leftarrow \sum_{j=1}^{2} P\left(v_{i, t}=l_{a} \mid v_{i, t-1}=l_{j}\right) P\left(v_{i, t-1}=l_{j}\right)$

where $P\left(v_{i}=l_{a}\right)$ is the probability that vehicle $i$ is traveling in lane $a$. This step is repeated for both vehicles, and then, the product of them is used to form the combined probabilities

$$
P\left(v_{1, t}=l_{a}, v_{2, t}=l_{b}\right)=P\left(v_{1, t}=l_{a}\right) P\left(v_{2, t}=l_{b}\right) .
$$

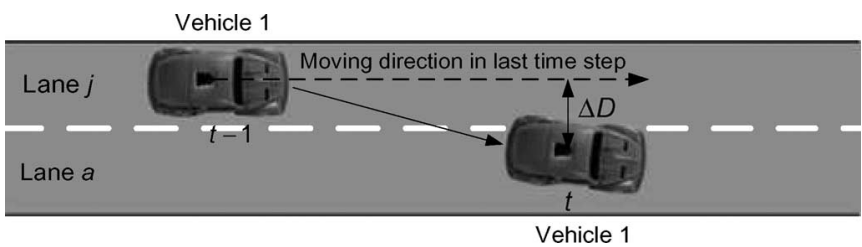

Fig. 2. Prediction step: This figure illustrates a vehicle (with an unknown position) switching lanes. The shortest distance $\Delta D$ from the position estimate at time $t$ to the line passing through the two most recent position estimates is used to calculate the conditional probability.

The conditional probabilities $P\left(v_{i, t}=l_{a} \mid v_{i, t-1}=l_{j}\right)$ for a vehicle are computed by comparing its current position estimate to the position at the last time step $t-1$. For example, by calculating the shortest distance $\Delta D$ from the vehicle position estimate from the GPS reading at time $t$ to the line passing through the two most recent position estimates, the probability at which vehicle 1 switches to lane $a$ (see Fig. 2) can be computed. In this case, by using zero as the mean value of the probability density function of a normal distribution $(1 / \sigma \sqrt{2 \pi}) e^{\left(-(\Delta D-0)^{2} / 2 \sigma^{2}\right)}$, if $\Delta D$ is close to zero, the probability at which vehicle 1 still stays in the same lane, i.e., vehicle 1 does not switch lane, is close to $100 \%$. In a similar fashion, one can develop all the 


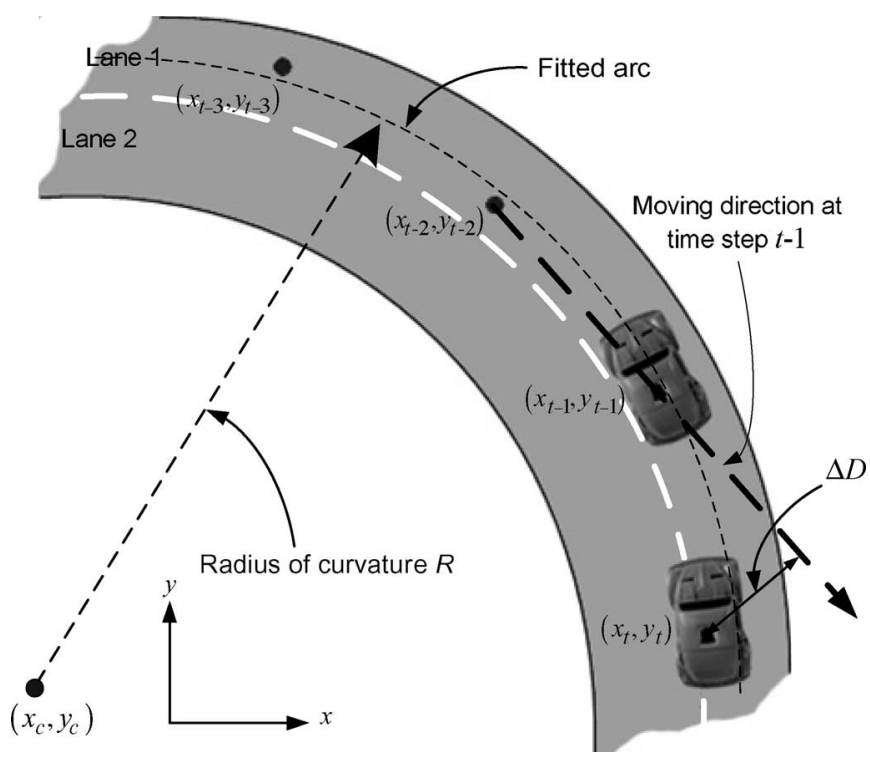

Fig. 3. Radius of curvature estimation: This figure illustrates the position estimates for a vehicle assumed to be traveling in lane 1 . The position estimates and moving direction of the vehicle are estimated from GPS data. The vehicle position estimates are not necessarily exactly in lane 1 , and the actual vehicle does not necessarily overlap with the position estimates, as shown in the figure.

conditional probabilities $P\left(v_{i, t}=l_{a} \mid v_{i, t-1}=l_{j}\right)$ that cover all possible situations.

It should be noted that a large $\Delta D$ can result from two possible situations: 1) the vehicle is switching lanes, or 2) the vehicle is simply following a curve in the road. For example, Fig. 3 shows that the vehicle is still in lane 1, but a large value of $\Delta D$ due to the curve would falsely indicate that the vehicle is switching to lane 2 .

To resolve this ambiguity, the lane-finding algorithm must estimate the radius of the lane curvature and compensate the drift caused by the curve. This can be done by using the least squares fitting algorithm based on successive vehicle positions. This algorithm allows us to fit an arc through the successive vehicle position estimates. When the vehicle changes lanes in the curved section of the road, its last position will not lie on the estimated arc, and the distance from the last position estimate to the arc will be used in the prediction step to predict if the vehicle is switching to the other lane. In our simulations and experiments, five successive vehicle position estimates were used to find the radius $R$ and center $\left(x_{c}, y_{c}\right)$ of the road curvature (see Fig. 4). The cost function to be minimized is

$$
S=\sum_{i=t-4}^{t} d_{i}^{2}=\sum_{i=t-4}^{t}\left(\sqrt{\left(x_{i}-x_{c}\right)^{2}+\left(y_{i}-y_{c}\right)^{2}}-R\right)^{2}
$$

where $\left(x_{i}, y_{i}\right)$ are the coordinates of the vehicle position estimate number $i$ (note that in (3), $i$ takes on values from $t-4$ to $t$ ). The following set of three equations can be solved for $R$, $x_{c}$, and $y_{c}$ using numerical methods:

$$
\left\{\begin{array}{l}
\frac{\partial S}{\partial x_{c}}=0 \\
\frac{\partial S}{\partial y_{c}}=0 \\
\frac{\partial S}{\partial R}=0 .
\end{array}\right.
$$

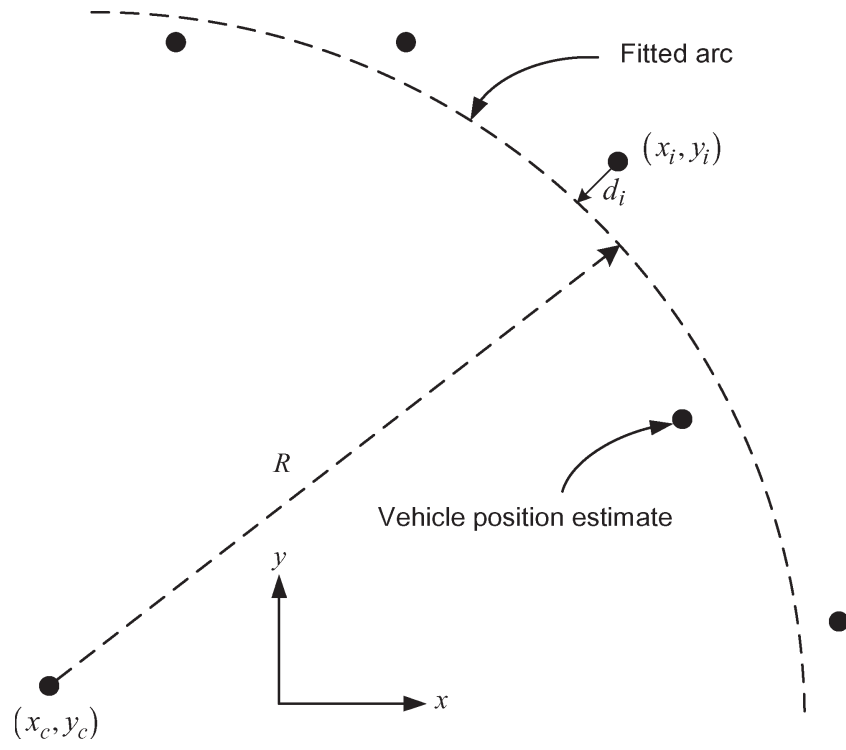

Fig. 4. Least squares method for approximating road curvature.

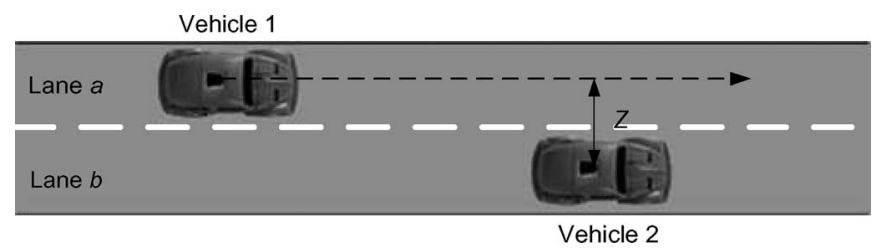

Fig. 5. Correction step: This figure illustrates two vehicles traveling on a highway. The distance $z$ is used to update the belief in prediction step.

\section{B. Correction}

Denote $z$ as the vehicle position estimates from GPS measurements that come in at time step $t$ for both vehicles, and $P\left(z \mid v_{1}=l_{a}, v_{2}=l_{b}\right)$ as the probability of perceiving $z$ when the two vehicles are in lanes $a$ and $b$, respectively. When the GPS measurements are taken into account, $P\left(z \mid v_{1}=l_{a}\right.$, $\left.v_{2}=l_{b}\right)$ is used to update the probability distribution at time $t$ according to Bayes' rule

$$
\begin{aligned}
P\left(v_{1, t}\right. & \left.=l_{a}, v_{2, t}=l_{b} \mid z_{t}\right) \\
\leftarrow & \frac{P\left(z_{t} \mid v_{1, t}=l_{a}, v_{2, t}=l_{b}\right) P\left(v_{1, t}=l_{a}, v_{2, t}=l_{b}\right)}{P\left(z_{t}\right)}
\end{aligned}
$$

where $P\left(z_{t}\right)$ has the purpose of normalizing the sum of all $P\left(v_{1, t}=l_{a}, v_{2, t}=l_{b} \mid z_{t}\right)$.

Suppose that at time $t$ a new measurement $z$ (see Fig. 5), which is the perpendicular distance from one vehicle to the other vehicle's direction of motion in this case, is available. The conditional probability $P\left(z \mid v_{1}=l_{a}, v_{2}=l_{b}\right)$ can be calculated based on $z$ using the probability density function $(1 / \sigma \sqrt{2 \pi}) e^{\left(-(z-\mu)^{2} / 2 \sigma^{2}\right)}$. The mean $\mu=w \times\left(l_{a}-l_{b}\right)$, where $w$ is the lane width. For example, in the case $l_{a}=2$ and $l_{b}=1$, the conditional probability $P\left(z \mid v_{1}=l_{a}, v_{2}=l_{b}\right)$ is closer to $100 \%$ when the measurement $z$ is closer to the lane width $w$.

The result for the two-vehicle-and-two-lane-road can be extended to the general case. Denote the number of vehicles that are communicating with each other as $n_{v}$, and the number of lanes as $n_{l}$. Let $P\left(v_{1, t}=l_{a}, v_{2, t}=l_{b}, \ldots, v_{n_{v}, t}=l_{x}\right)$ be 


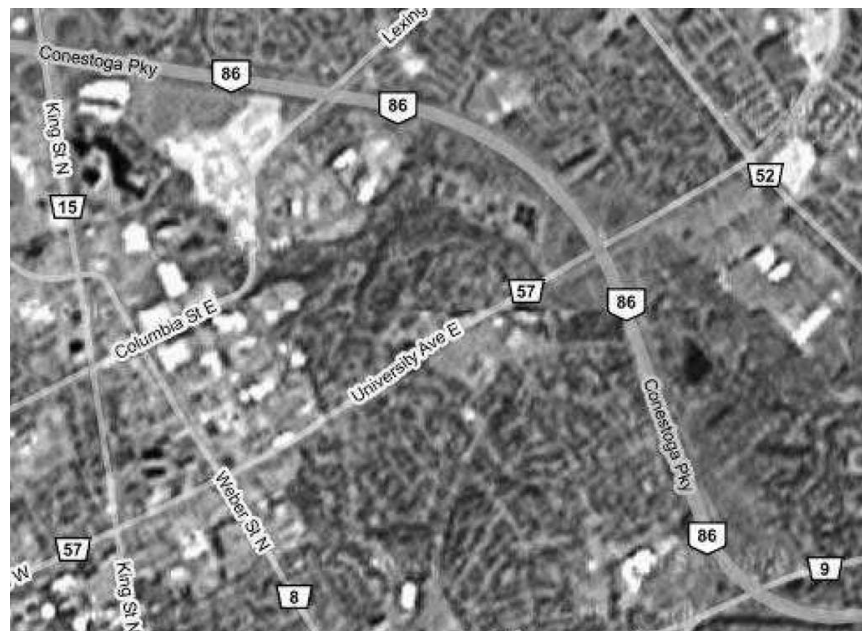

Fig. 6. Highway 86, Waterloo, ON, Canada.

the probability that vehicle 1 is traveling in lane $a$, vehicle 2 is in lane $b$, etc., at time $t$, where $a, b, \ldots, x$ take on values between 1 and $n_{l}$. No initial estimates of the vehicle lane positions are required, and the initial probabilities are equally set to $1 /\left(n_{v} \times n_{l}\right)$. The probability distribution at time $t$ for the prediction step is given by

$$
\begin{aligned}
P\left(v_{i, t}=\right. & \left.l_{a}\right) \leftarrow \sum_{j=1}^{n_{l}} P\left(v_{i, t}=l_{a} \mid v_{i, t-1}=l_{j}\right) P\left(v_{i, t-1}=l_{j}\right) \\
P\left(v_{1, t}=\right. & \left.l_{a}, v_{2, t}=l_{b}, \ldots, v_{n_{v}, t}=l_{x}\right) \\
& =P\left(v_{1, t}=l_{a}\right) P\left(v_{2, t}=l_{b}\right), \ldots, P\left(v_{n_{v}, t}=l_{x}\right) .
\end{aligned}
$$

Bayes' rule for the correction step is

$$
P\left(v_{1, t}=l_{a}, v_{2, t}=l_{b}, \ldots, v_{n_{v}, t}=l_{x} \mid z_{t}\right) \leftarrow \frac{Q S}{P\left(z_{t}\right)}
$$

where $Q=P\left(z_{t} \mid v_{1, t}=l_{a}, v_{2, t}=l_{b}, \ldots, v_{n_{v}, t}=l_{x}\right)$, and $S=$ $P\left(v_{1, t}=l_{a}, v_{2, t}=l_{b}, \ldots, v_{n_{v}, t}=l_{x}\right)$.

With the developed Markov-based algorithm, we are able to implement simulations and experiments, which will be discussed in Sections IV and V.

\section{Simulation Results}

To implement our simulations, the VISSIM software package [27] was used. VISSIM is a microscopic, time-step, and behavior-based simulator that is developed to analyze the full range of functionally classified roadways. It is capable of modeling traffic with various control measures in a 3-D environment. VISSIM lets us communicate and control the behaviors of vehicles through a dynamic link library (DLL) file compiled from $\mathrm{C} / \mathrm{C}++$ code. Vehicle parameters from the external driver model DLL output function are stored within member variables of a designated vehicle class object.

For a precise analysis of the algorithm, a map of a road based on an actual highway, as shown in Fig. 6, was built in VISSIM, and a number of simulations with different situations were implemented. In all simulations, the GPS data are modeled by adding a random walk bias of maximum amplitude of $3 \mathrm{~m}$ and

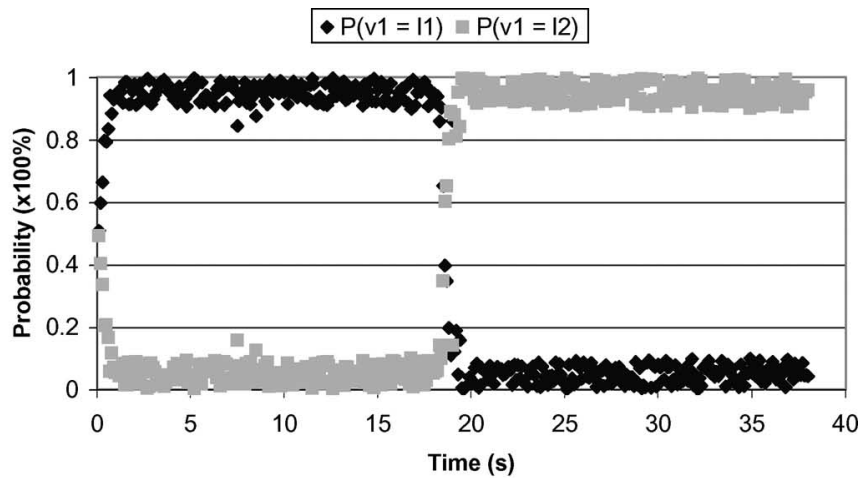

(a)

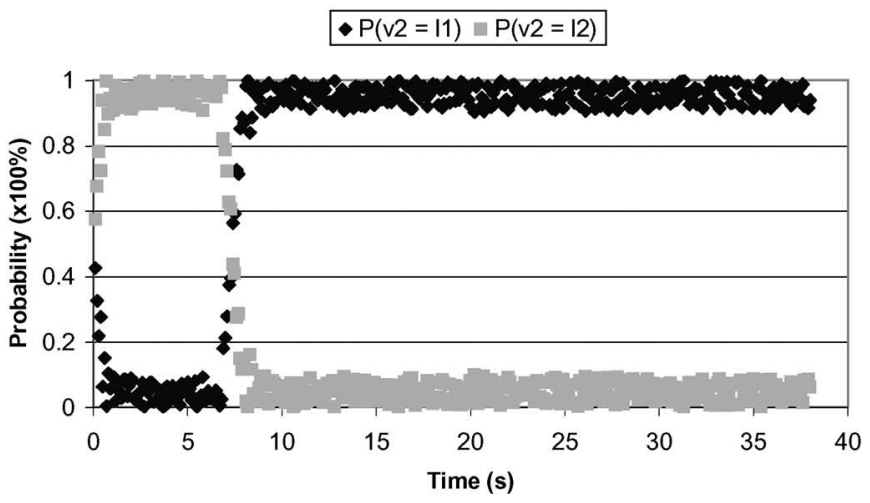

(b)

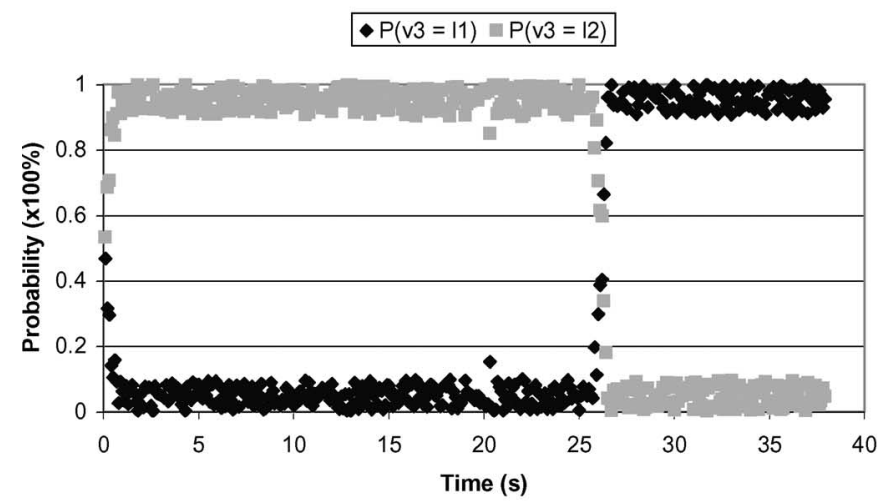

(c)

Fig. 7. Probability distribution for simulation of three cars (car 1 to car 3 from top to bottom) on a two-lane highway.

Gaussian noise having a standard deviation of $0.5 \mathrm{~m}$ to the position of each vehicle.

Figs. 7 and 8 show a simulation with three cars and a twolane road. In this simulation, no position filters are used. The scenario is as follows: Initially, car 1 is in lane 1 , and car 2 and car 3 are both in lane 2 . After $6 \mathrm{~s}$, car 2 switches to lane 1 . Car 1 switches to lane 2 after $16 \mathrm{~s}$, and car 3 moves to lane 1 after $26 \mathrm{~s}$ (Fig. 8). It can be seen that the algorithm accurately estimates the lane positions of the vehicles. The corresponding probabilities are given in Fig. 7. The algorithm also works when vehicles have frequent oscillatory weaving actions within their lanes (see Fig. 9), although this rarely happens on real highway systems due to safety concerns. Fig. 9 shows the lane estimation results without position filtering for three cars on a two-lane highway. It can be seen that car 1 and car 3 have wrong lane 

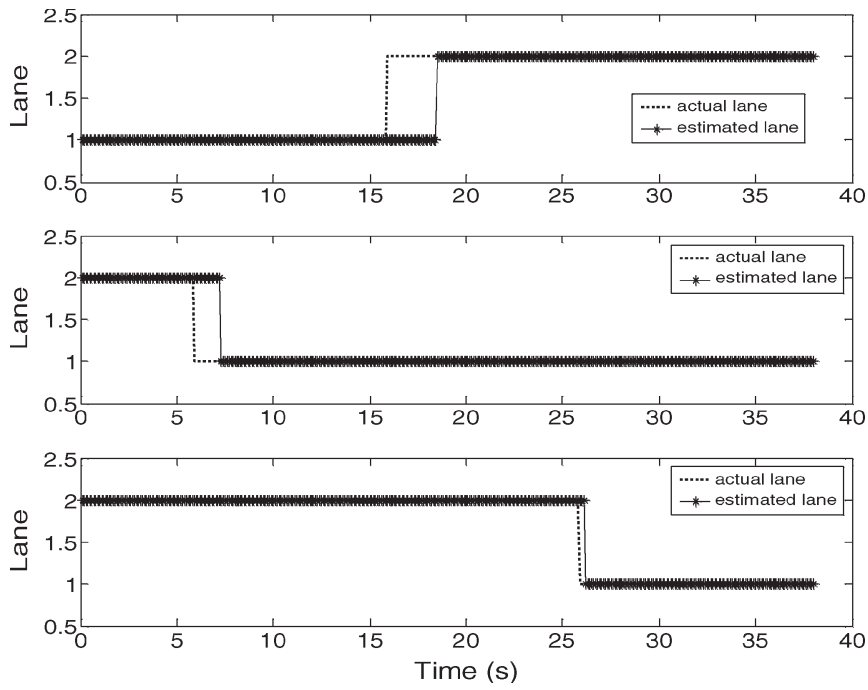

Fig. 8. Simulation result for three cars (car 1 to car 3 from top to bottom) on a two-lane highway.
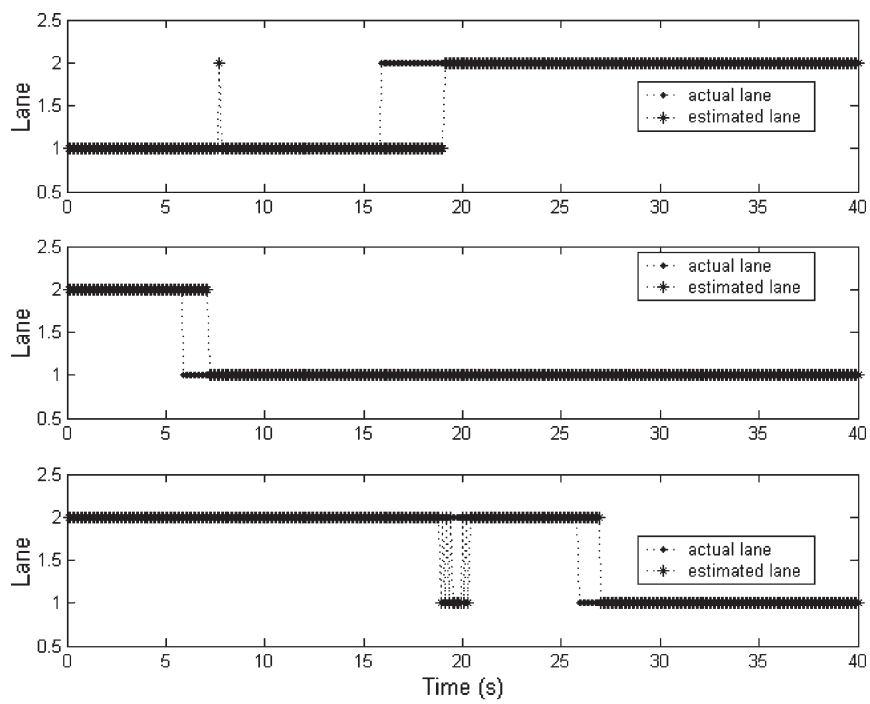

Fig. 9. Impact of oscillatory weaving (car 1 to car 3 from top to bottom): This figure shows a simulation in which car 1, car 2, and car 3 follow sinusoidal paths with different amplitudes $(-1,-1$, and $1 \mathrm{~m})$ and frequencies $(1 / 4,1 / 4.5$, and $1 / 5 \mathrm{~Hz}$ ).

estimation by only a small fraction of time. The improved results when position filters consisting of a low-pass Butterworth filter and a particle filter for all three vehicles are added to reject the modeled GPS receiver noise (Gaussian noise) are shown in Fig. 10. The speeds of the vehicles in all simulations are $80-100 \mathrm{~km} / \mathrm{h}$. Another case of interest is when the vehicles frequently change their lanes. The convergence rate of the algorithm in this case is faster since the localization algorithm has more frequent information update for both prediction and correction steps.

Importantly, since the lane positions of vehicles are determined relative to each other, it is not necessary to know the number of lanes for the algorithm to work. The lowest lane position is always lane 1 , and the other lanes are numbered relative to the first lane. In practice, as the number of vehicles increase, the algorithm will be able to estimate the lane positions faster
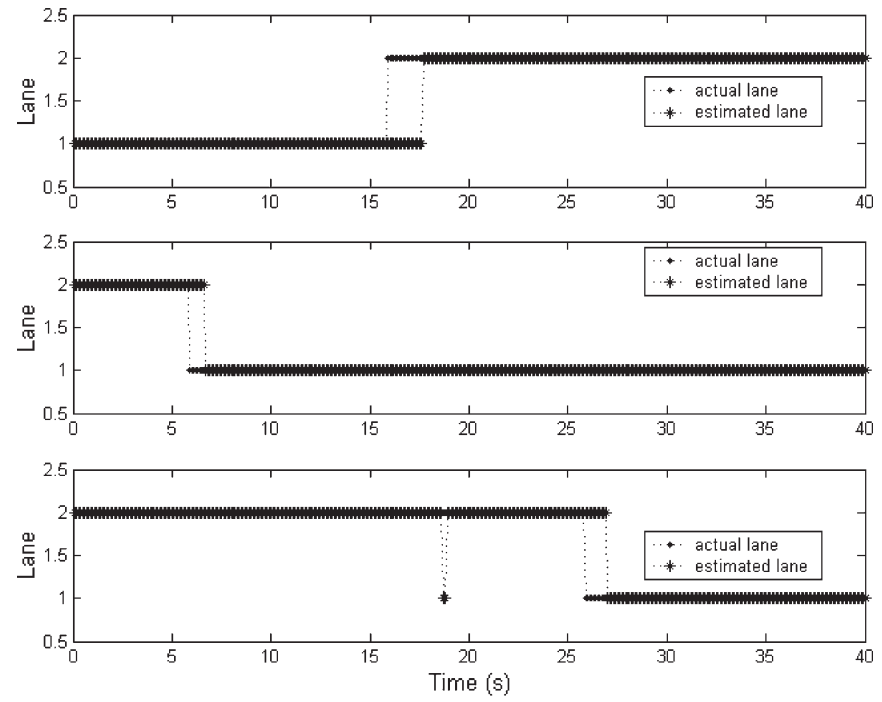

Fig. 10. Improved lane estimation with position filters (car 1 to car 3 from top to bottom).

TABLE I

Percentage of Time With Correct Lane ESTIMATION FOR SIMULATIONS

\begin{tabular}{|l|l|}
\hline Simulation & Correct lane estimation (\%) \\
\hline $\begin{array}{l}\text { No oscillatory weaving, } \\
\text { no position filters }\end{array}$ & Car 1: 93.1\% \\
& Car 2: $96.3 \%$ \\
& Car 3: $98.9 \%$ \\
\hline With oscillatory weaving, & Car 1: 91.8\% \\
no position filters & Car 2: $96.8 \%$ \\
& Car 3: $94.5 \%$ \\
\hline With oscillatory weaving and & Car 1: 95.5\% \\
position filters & Car 2: $98.1 \%$ \\
& Car 3: $96.7 \%$ \\
\hline
\end{tabular}

and more reliably. The percentage of time with correct lane estimation for the simulations are summarized in Table I.

\section{EXPERIMENTAL RESUlTS}

To validate the algorithm, a number of experiments with different scenarios were implemented on the same highway. Real GPS data were collected for the two cars used in the test. The approximately 4-km-highway section shown in Fig. 6 was used to conduct the experiments. The speeds of the vehicles used in the tests were between 90 and $110 \mathrm{~km} / \mathrm{h}$.

One of those experiments is shown in Figs. 11 and 12. These figures show the lane estimation results without using position filters to demonstrate the effect of GPS measurement noise. The strategy was as follows: Initially, car 1 was in lane 1 , and car 2 was in lane 2. After $22 \mathrm{~s}$, car 1 switched to lane 2. Car 2 switched to lane 1 right after car 1 completed its lane changing. Car 1 moved back to lane 1 after $40 \mathrm{~s}$ and finally moved to lane 2 after $60 \mathrm{~s}$. Car 2 switched back to lane 2 at the fortieth second and maintained its lane position until the end of the experiment.

In this experiment, two cars equipped with low-cost GPS receivers with different sampling rates and variances drove on the highway. The GPS receiver for the first car was a Garmin 18-5, which has a sampling rate of $5 \mathrm{~Hz}$. The GPS receiver for the second car was a LocSense 40-CM, whose 


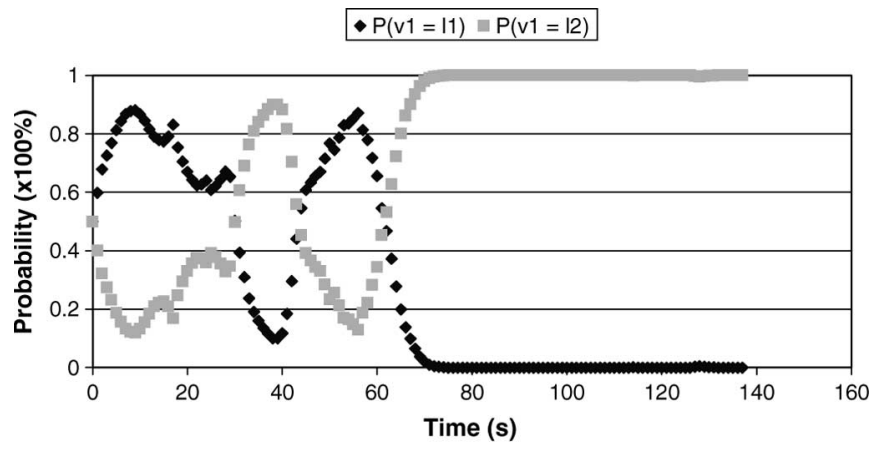

(a)

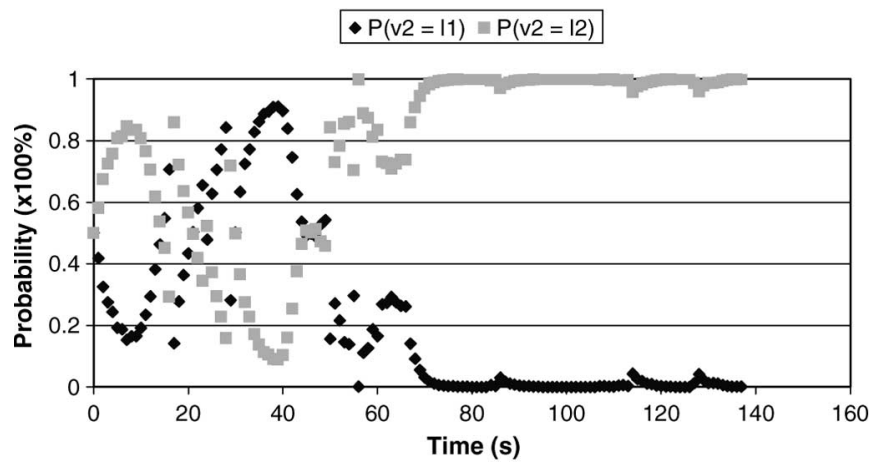

(b)

Fig. 11. Probability distribution for an experiment without GPS measurement filtering.
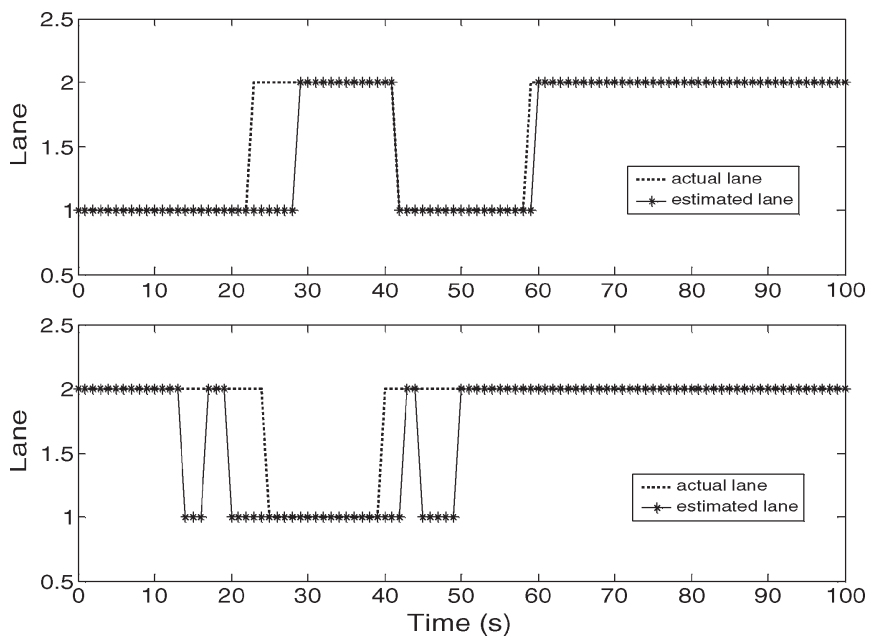

Fig. 12. Lane estimation result for two cars (car 1 to car 2 from top to bottom) on a highway without GPS measurement filtering.

sampling rate is $1 \mathrm{~Hz}$. Both GPS receivers output National Marine Electronics Association 0183 standard messages. The second GPS receiver signal has a much higher noise when compared to the first receiver. This resulted in probability distributions with higher variability, as shown in Fig. 11. The performance of lane position estimation is thus reduced in that the lane the vehicle occupied was not always accurately predicted (see Fig. 12).

Figs. 13 and 14 show the effects of the improved noise rejection on the probability and lane estimates when a position filter for car 2 was used in comparison to the results shown

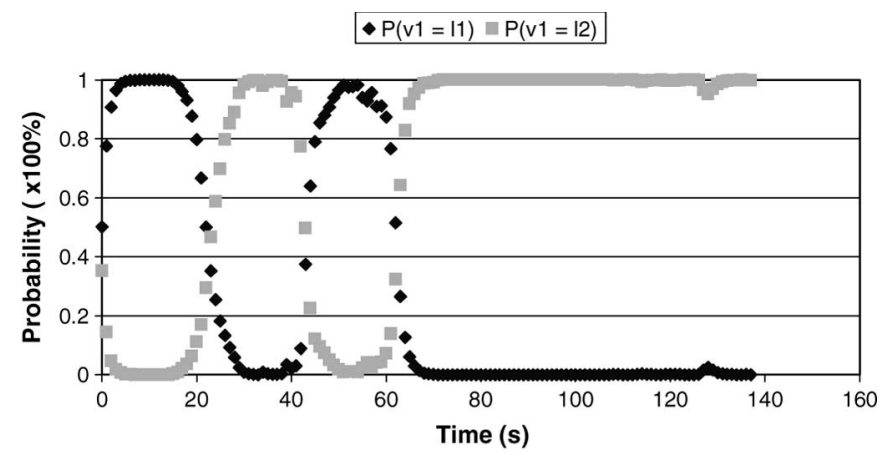

(a)

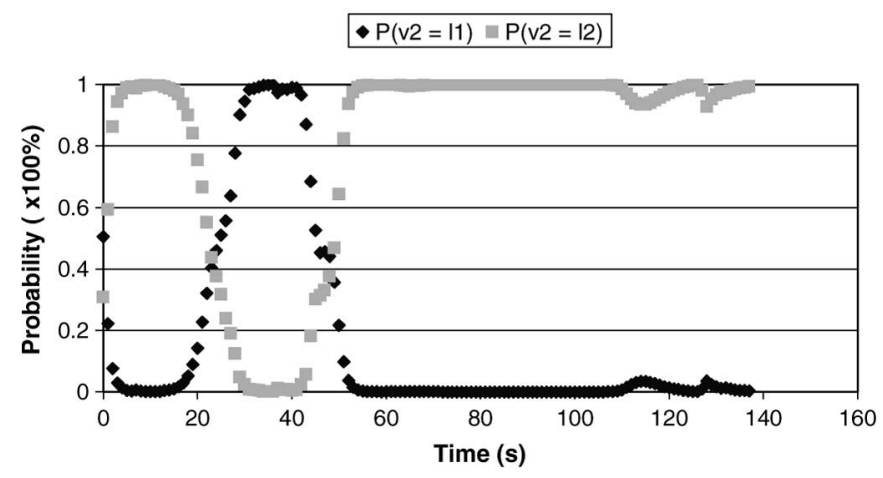

(b)

Fig. 13. Improved probability distribution for an experiment with GPS measurement filtering.
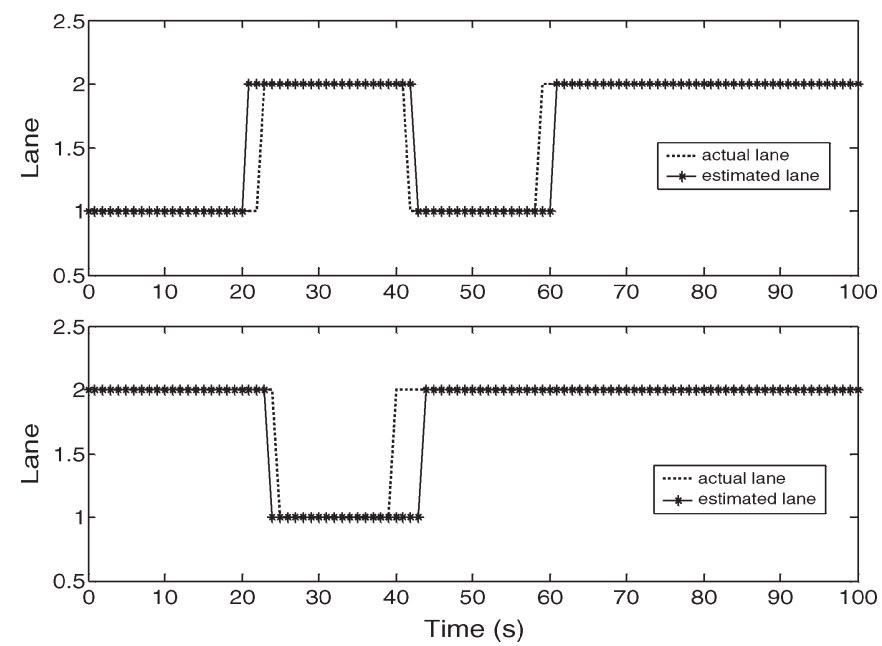

Fig. 14. Improved results with GPS measurement filtering.

in Figs. 11 and 12. The GPS data for car 2 before and after being filtered are shown in Fig. 15. This filtering resulted in more accurate and faster estimates of lane position. This improvement indicates that, in practice, low-cost GPS receivers can be effectively fused with a filter to obtain low-noise GPS measurements, rather than resorting to the use of more expensive sensors. It is also interesting to point out that the algorithm sometimes could anticipate the lane-changing action before the vehicles completely moved to the other lanes, as indicated in Fig. 14, at around the twenty-second second. This can be explained by the fact that when a vehicle starts making a lane change maneuver, its lateral displacement notifies the 


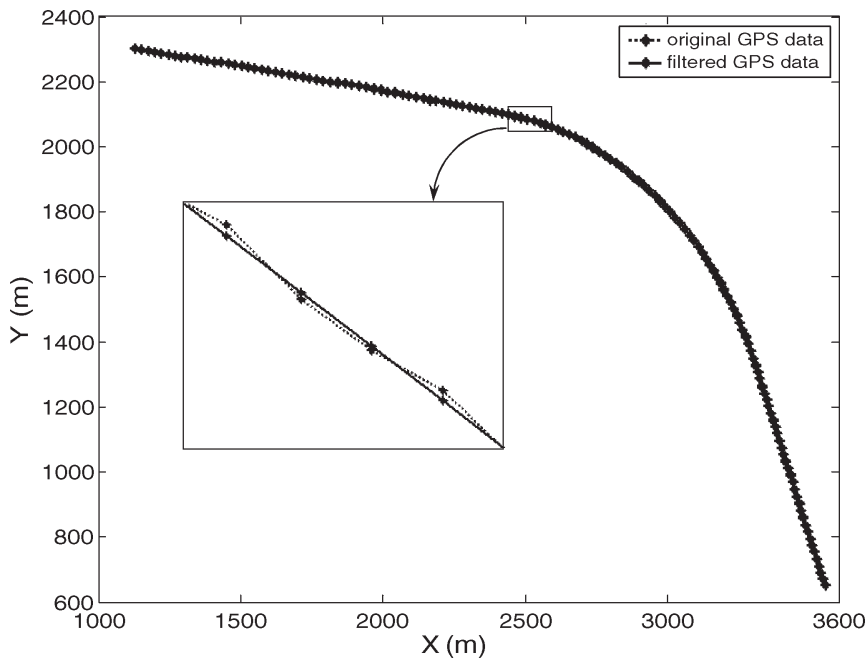

Fig. 15. Original GPS data versus filtered GPS data for car 2.

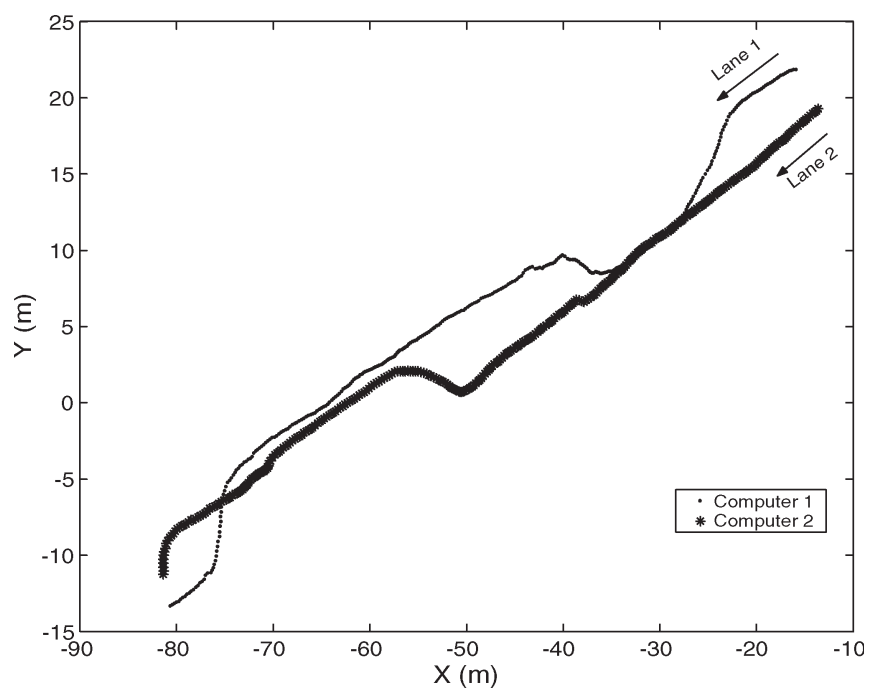

Fig. 16. GPS measurements at walking speed.

prediction step of the localization algorithm that the vehicle is about to make a lane change before the lane changing is completed.

To demonstrate that the algorithm can work effectively at low speeds, an experiment with two walking persons holding two laptops equipped with two Garmin 18 GPS receivers was conducted on Wilhelm Street, a two-lane road in Kitchener, ON, Canada. The walking speeds for both persons were approximately $4 \mathrm{~km} / \mathrm{h}$ (estimated from GPS data). The GPS measurements for both computers are plotted in Fig. 16. The resulting probability distributions and estimated lane positions are shown in Figs. 17 and 18, respectively.

Table II summarizes the percentage of time with correct lane estimation for the experiments.

\section{CONCLUSION}

A new lane position estimation algorithm that uses a Markov-based approach based on cooperative driving models has been proposed in this paper. The key idea of Markov

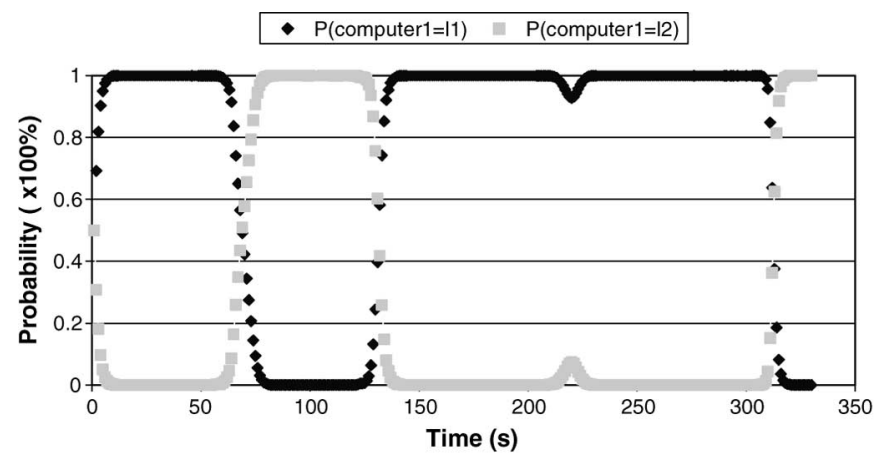

(a)

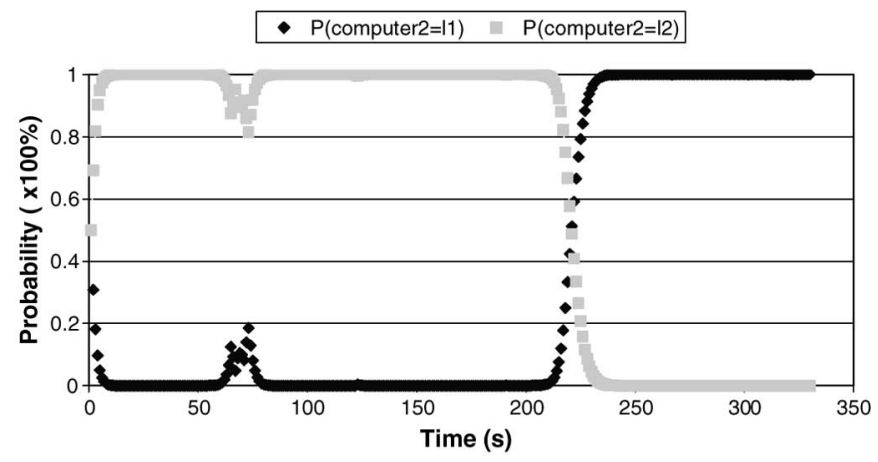

(b)

Fig. 17. Probability distribution for two laptops at walking speed.
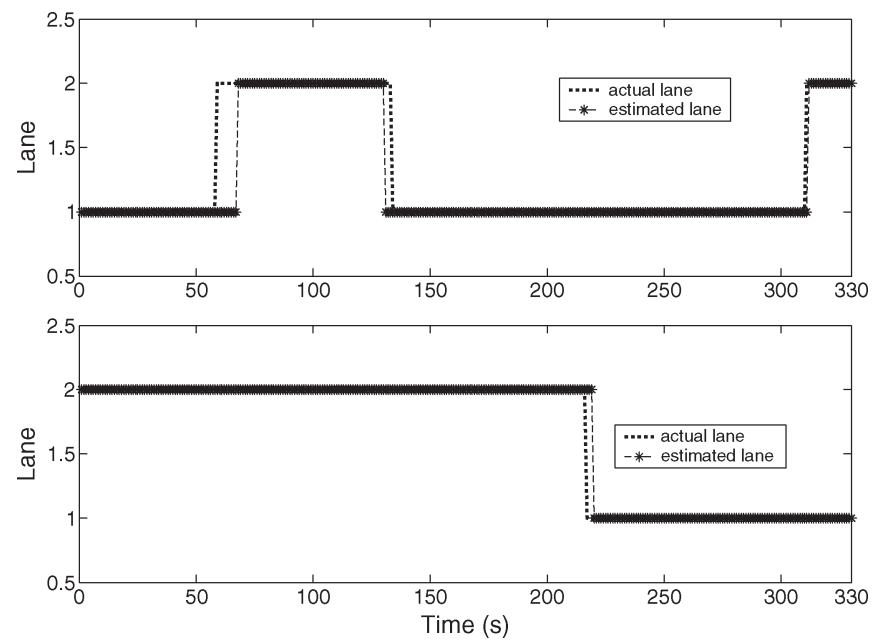

Fig. 18. Estimated lane positions for two laptops at walking speed.

TABLE II

Percentage of Time With Correct Lane ESTIMATION FOR EXPERIMENTS

\begin{tabular}{|l|l|}
\hline Experiment & Correct lane estimation (\%) \\
\hline Two cars without position filters & Car 1: 93.0\% \\
& Car 2: 86.0\% \\
\hline Two cars with position filters & Car 1: 95.0\% \\
& Car 2: 95.0\% \\
\hline Two laptops at walking speed & Computer 1: $96.0 \%$ \\
& Computer 2: $99.0 \%$ \\
\hline
\end{tabular}

localization is to maintain a probability density over the space of all lane positions of a vehicle on a highway. Since the algorithm needs no prior knowledge of the vehicle's initial lane 
position, it is possible to globally localize the vehicle from scratch and to recover from localization failures or GPS outage in which the positions of the vehicle are lost.

In comparison to conventional lane-positioning methods, which usually deal with complicated image processing techniques and/or expensive equipment, the proposed method only requires low-cost GPS receivers, IVC, and a simple localization algorithm. Simulation and experimental results have shown the efficiency of the algorithm, even when the GPS data are significantly degraded.

The limitation of the proposed strategy lies in the fact that it only uses GPS data to estimate lane positions. This might be challenging where GPS data are not available or GPS signal is completely blocked by large obstacles like in a long tunnel. One possible solution to this problem is to fuse the GPS data with another type of sensor, such as an IMU, until GPS data are again available.

Future research will continue with more complicated situations such as determining lane position on highways with intersections. Issues to be addressed include derivation of per-lane conditional probability models that are required at intersections. For example, the positioning system may indicate that $100 \%$ of drivers in the left lane at a particular intersection turn left, $50 \%$ of drivers in the right lane go straight, and 50\% turn right.

\section{REFERENCES}

[1] J. Du, J. Masters, and M. Barth, "Lane-level positioning for in-vehicle navigation and automated vehicle location (AVL) systems," in Proc. IEEE Intell. Transp. Syst. Conf., Washington DC, 2004, pp. 35-40.

[2] S. S. Ieng, J. Vrignon, D. Gruyer, and D. Aubert, "A new multi-lane detection using multi-camera for robust vehicle location," in Proc. IEEE Intell. Veh. Symp., Las Vegas, NV, 2005, pp. 700-705.

[3] J. C. McCall and M. M. Trivedi, "Video-based lane estimation and tracking for driver assistance: Survey, system, and evaluation," IEEE Trans. Intell. Transp. Syst., vol. 7, no. 1, pp. 20-37, Mar. 2006.

[4] G. Pierre-Yves and K. Jeff, "Enhanced navigation system for road telematics," in Proc. 3rd Swiss Transp. Res. Conf., 2003, pp. 1-16.

[5] U.S. Department of Transportation, Enhanced Digital Mapping Project Final Report, 2004, Washington DC.

[6] J. Wang, S. Schroedl, K. Mezger, R. Ortloff, A. Joos, and T. Passegger, "Lane keeping based on location technology," IEEE Trans. Intell. Transp. Syst., vol. 6, no. 3, pp. 351-356, Sep. 2005.

[7] K. Y. Chiu and S. F. Lin, "Lane detection using color-based segmentation," in Proc. IEEE Intell. Veh. Symp., 2005, pp. 706-711.

[8] B. D. Stewart, I. Reading, M. S. Thomson, T. D. Binnie, K. W. Dickinson, and C. L. Wan, "Adaptive lane finding in road traffic image analysis," in Proc. 7th IEEE Int. Conf. Road Traffic Monitoring Control, London, U.K., 1994, pp. 133-136.

[9] J. Gangyi, C. Yanhua, Y. Mei, and Z. Yi, "Approach to lane departure detection," in Proc. IEEE 5th ICSP, 2000, pp. 971-974.

[10] A. H. S. Lai and N. H. C. Yung, "Lane detection by orientation and length discrimination," IEEE Trans. Syst., Man, Cybern. B, Cybern., vol. 30, no. 4, pp. 539-548, Aug. 2000.

[11] T. K. Lim and M. A. Do, "Camera detection, classification, and positioning of vehicles on a multi-lane road," in Proc. IEEE Singapore Int. Conf. Inf. Eng., Singapore, 1993, vol. 1, pp. 374-378.

[12] M. M. Artimy, W. Robertson, and W. J. Phillips, "Connectivity in intervehicle ad-hoc networks," in Proc. Eng. Can. Conf. Electr. Comput., May 2004, vol. 1, pp. 293-298.

[13] J. Blum, A. Eskandarian, and L. Hoffman, "Performance characteristics of inter-vehicle ad hoc networks," in Proc. IEEE 6th Int. Conf. Intell. Transp. Syst., Shanghai, China, 2003, pp. 114-119.

[14] Z. D. Chen, H. T. Kung, and D. Vlah, "Ad hoc relay wireless networks over moving vehicles on highways," in Proc. 2nd ACM Int. Symp. Mobile Ad Hoc Netw. Comput., 2001, pp. 247-250.

[15] W. Enkelmann, "FleetNet applications for inter-vehicle communication," in Proc. IEEE Intell. Veh. Symp., Columbus, OH, 2003, pp. 162-167.
[16] Standard specification for telecommunications and information exchange between road-side and vehicle systems $-5 \mathrm{GHz}$ band dedicated shortrange communications (DSRC) medium access control (MAC) and physical layer (PHY) specifications, ASTM E2213-03, Jul. 2003.

[17] C. E. Palazzi, S. Ferretti, M. Roccetti, G. Pau, and M. Gerla, "How do you quickly choreograph inter-vehicular communications? A fast vehicleto-vehicle multi-hop broadcast algorithm, explained," in Proc. 3rd IEEE CCNC/NIME, Jan. 2007, pp. 960-964.

[18] I. Berger, Standards for Car Talk, Mar. 2007. [Online]. Available: www. theinstitute.ieee.org

[19] M. S. Grewal, L. R. Weill, and A. P. Andrews, Global Positioning Systems, Inertial Navigation, and Integration. Hoboken, NJ: Wiley, 2000.

[20] I. M. Rekleitis, A Particle Filter Tutorial for Mobile Robot Localization TR-CIM-04-02. Montreal, QC, Canada: Centre Intell. Mach., McGill Univ., 2003.

[21] S. Thrun, W. Burgard, and D. Fox, Probabilistic Robotics. Cambridge, MA: MIT Press, 2005.

[22] S. Thrun, "Particle filters in robotics," in Proc. 17th Annu. Conf. UAI, 2002, pp. 511-518.

[23] B. A. Shenoi, Introduction to Digital Signal Processing and Filter Design Hoboken, NJ: Wiley, 2005.

[24] S. J. Russell and P. Norvig, Artificial Intelligence: A Modern Approach. Englewood Cliffs, NJ: Prentice-Hall, 1995.

[25] D. Fox, "Markov localization: A probabilistic framework for mobile robot localization and navigation," Ph.D. dissertation, Dept. Comput. Sci., Univ. Bonn, Bonn, Germany, Dec. 1998.

[26] S. Koenig and R. Simmons, "A robot navigation architecture based on partially observable Markov decision process models," in AI-ROBOTS, D. Kortenkamp et al., Ed. Cambridge, MA: MIT Press, 1998.

[27] VISSIM. [Online]. Availhttp://www.english.ptv.de/cgi-bin/traffic/traf vissim.pl

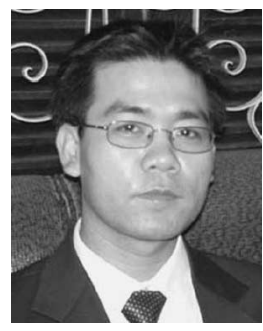

Thanh-Son Dao received the M.Sc. degree in mechanical engineering from Dayeh University, Changhua, Taiwan, R.O.C, in 2005. He is currently working toward the Ph.D. degree in mechatronics with the Laboratory for Autonomous and Intelligent Robotics, Department of Mechanical and Mechatronics Engineering, University of Waterloo, Waterloo, ON, Canada.

His research interests include intelligent vehicles, dynamic collaborative driving, and autonomous vehicle networking.

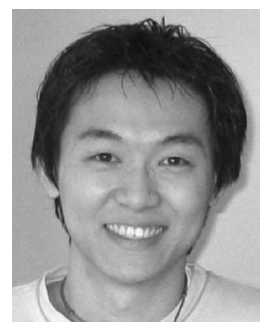

Keith Yu Kit Leung received the B.A.Sc. degree in mechanical engineering from the University of Waterloo, Waterloo, ON, Canada. He is currently working toward the M.A.Sc. degree in mechatronics and mechanical engineering with the Laboratory for Autonomous and Intelligent Robotics, Department of Mechanical and Mechatronics Engineering, University of Waterloo, under the supervision of Dr. C. Clark and Dr. J. Huissoon.

His research interest is in the field of mobile robotics, with particular interests in autonomous localization and multirobot systems. Some projects that he has been involved with in the past include the development of a microscopic traffic simulator for intervehicle communication research and the development of an autonomous vehicle. 


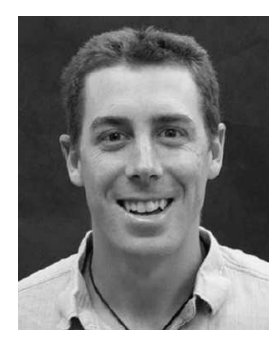

Christopher Michael Clark (M'04) received the $\mathrm{Ph} . \mathrm{D}$. degree from Stanford University, Stanford, $\mathrm{CA}$, in 2004.

He was an Assistant Professor with the University of Waterloo, Waterloo, ON, Canada, from 2004 to 2007, where he developed the Laboratory for Autonomous and Intelligent Robotics. He is currently an Assistant Professor with the Department of Computer Science, California Polytechnic State University, San Luis Obispo. His research areas include motion planning, multirobot systems, intelligent transportation systems, modular and reconfigurable robots, and underwater robot systems.

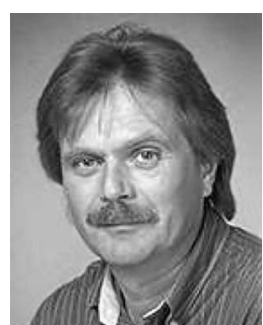

Jan Paul Huissoon received the Ph.D. degree in mechanical engineering from the University of Dublin, Trinity College, Dublin, Ireland, in 1983.

In 1986, he joined the University of Waterloo, Waterloo, ON, Canada, where he is currently the Director of the Mechatronics Engineering Program. His research interests include robotic welding and seam tracking, autonomous robotics, mechatronic design, modeling, and control.

Dr. Huissoon is a member of the Institute of Engineers of Ireland and the American Welding Society and a Senior Member of the Society of Manufacturing Engineers. He is a Registered Professional Engineer. 